\title{
Atherogenic index of plasma is a novel and better biomarker associated with obesity: a population-based cross-sectional study in China
}

Xiaowei Zhu ${ }^{1,2+}$, Lugang Yu ${ }^{3+}$, Hui Zhou ${ }^{3}$, Qinhua Ma ${ }^{4}$, Xiaohua Zhou ${ }^{4}$, Ting Lei ${ }^{3}$, Jiarong Hü ${ }^{4}$, Wenxin Xu ${ }^{4}$, Nengjun $\mathrm{Yi}^{5}$ and Shufeng Lei $\mathrm{i}^{1,2^{*}}$

\begin{abstract}
Background: Atherogenic index of plasma (AIP) has been reported to be associated with cardiovascular diseases. However no study has yet systematically evaluated the association between AIP and obesity and its advantage in obesity prediction compared with conventional lipid components.

Methods: A total of 6465 participants aged over 30 years were included in this study. Blood lipid components including triglyceride $(\mathrm{TG})$, total cholesterol (TC), high-density lipoprotein cholesterol (HDL-C) and low-density lipoprotein cholesterol (LDL-C) were measured, and AIP was calculated as $\log _{10}(T G / H D L-C)$. Pearson correlation analyses, multivariable logistic analyses and predictive analyses were used to evaluate the association and discrimination ability between AIP, four conventional lipid profiles and obesity.
\end{abstract}

Results: Subjects in the higher quartiles of AIP all had a significantly increased risk of obesity compared with those in the lowest quartile ( $P$ for trend< 0.01 ). AlP showed a stronger association with obesity than the conventional lipid components as the pearson coefficient reached up to 0.372 and the adjusted odds ratio was 5.55. Using AIP rather than HDL-C and TG significantly improved risk prediction for obesity (AUC improvement $=0.011, P=0.011$; Continuous net reclassification index $=29.55 \%, P<0.01$; Category net reclassification index $=6.06 \%$; Integrated discrimination improvement $=0.68 \%, P<0.01$ ).

Conclusions: Higher AIP level was positively and strongly associated with obesity. AIP is a novel and better biomarker associated with obesity. Controlling the AIP level would be more helpful for the prevention of obesity.

Keywords: Obesity, Atherogenic index of plasma, Blood lipid components

\section{Background}

Obesity has become a global problem of public health over the past few decades with its high prevalence (range between $11 \%$ and $15 \%$ ) and medical burden in both developed and developing countries [1]. As generally known, obesity is considered as a medical condition in which

\footnotetext{
*Correspondence: leisf@suda.edu.cn

${ }^{\dagger}$ Equal contributors

${ }^{1}$ Center for Genetic Epidemiology and Genomics, School of Public Health, Medical College of Soochow University, Suzhou, Jiangsu 215123, China

${ }^{2}$ Jiangsu Key Laboratory of Preventive and Translational Medicine for Geriatric Diseases, School of Public Health, Medical College of Soochow University, Suzhou, Jiangsu 215123, China

Full list of author information is available at the end of the article
}

excess body fat has been accumulated. The storage room of body fat that adipocytes and adipose tissue also store the greatest amount of body lipids including triglycerides and free cholesterol [2]. Recent years, most studies have reported that blood lipids and obesity are closely related [3-5]. Looking for the optimal blood lipid index to be used as a novel biomarker for obesity will make up for the limitation of body mass index (BMI) acted as a simple anthropometric indicator of obesity. The lipid biomarker as a direct biological target will also be helpful in obesity prevention and therapeutic treatment.

Atherogenic index of plasma (AIP) is a novel index composed of triglycerides and high-density lipoprotein 
cholesterol [6]. It has been used to quantify blood lipid levels and commonly used as optimal indicator of dyslipidemia and associated diseases (e.g., cardiovascular diseases) [7-9]. However, no published study has yet examined the association between AIP and obesity, and especially the discrimination ability between AIP, conventional lipids and obesity risk. Our study thus aimed to assess the association between AIP, the conventional lipid components and obesity in a large-scale Chinese population and further systematically highlighted the predictive advantage of AIP for obesity compared with the conventional lipid components.

\section{Methods}

\section{Study participants}

The study subjects were recruited via a local health physical examination program conducted in two municipal districts of Suzhou in southeast of China, during June 2013 to December 2013. Participants enrolled in the study met the criteria of age over 30 years and Chinese Han ethnicity. After excluding subjects for lacking blood samples, a total of 6465 subjects were finally included in the analysis. The study was approved by the ethical committee of Soochow University. Subjects agreeing to participate into the present study provided a written informed consent.

\section{Data collection}

Health examination was performed in the morning after the examinees fasted overnight. Anthropometric indices were measured by an eligible physician. Weight was measured in light indoor clothing without shoes, using a calibrated balance beam scale, and height was measured using a calibrated stadiometer. Body mass index (BMI) was calculated as body weight (in kilograms) divided by the square of height (in meters). Obesity was defined as $\mathrm{BMI} \geq 28 \mathrm{~kg} / \mathrm{m}^{2}$ according to the criteria of China Obesity Task Force [10]. Seated blood pressure was measured by mercury sphygmomanometer after 10 -min rest in the sitting room.

Peripheral blood was drawn into an EDTA-containing tube and stored into a $4{ }^{\circ} \mathrm{C}$ refrigerator, and was subjected to biochemical experiments within $24 \mathrm{~h}$. Glucose oxidase method (Homa, Beijing, China) was used to detect fasting blood glucose (FBG). Blood lipid indexes including triglyceride (TG), total cholesterol (TC), highdensity lipoprotein cholesterol (HDL-C) and low-density lipoprotein cholesterol (LDL-C) were measured by Hitachi 7180 autoanalyzer (Hitachi, Tokyo, Japan). Atherogenic index of plasma (AIP) was calculated as logarithmic transformation of the ratio of TG to HDL-C.

\section{Statistical analysis}

All participants were divided into obesity and non-obesity groups as well as the quartile groups based on their AIP values $(<-0.231,-0.231$ to $-0.052,-0.053$ to 0.146 , $\geq 0.147$ ). The baseline variables were compared using the Student's t-test, one-way ANOVA, or the chi-squared test, as appropriate. The Pearson correlation analyses were used to assess the relationship between AIP, four lipid components and $\mathrm{BMI}$ in total participants.

Univariate and multivariate logistic regression analyses were conducted to evaluate the association between AIP, four lipid components and obesity. Total participants were further categorized into four groups according to the quartiles of AIP. The odds ratio (OR) along with its 95\% confidence interval $(95 \% \mathrm{CI})$ of obesity were estimated for higher three categories of AIP with the lowest one as a reference. A linear trend in the ORs of obesity was performed by entering AIP quartiles as continuous parameters in the model. Area under the curve (AUC) of receiver operating characteristic (ROC), net reclassification index (NRI) and integrated discrimination improvement (IDI) were calculated to compare the predictive value between AIP and conventional lipid profiles including TG and HDL for predicting obesity. All statistical tests were two-tailed and were considered significant for $p$ less than 0.05. Statistical analyses were performed using SAS statistical software (version 9.4, SAS Institute, Cary, NC, USA) and R software (version 3.0, the R Foundation for Statistical Computing, Vienna, Austria).

\section{Results}

Among 6465 participants included in the study, 503 of them $(7.78 \%)$ were obesity. As shown in Table 1, participants with obesity were more likely to have higher systolic blood pressure (SBP), diastolic blood pressure (DBP), FBG, TG, TC, LDL-C and AIP as well as a lower level of HDL-C. In Table 2, the participants with higher AIP

Table 1 Baseline Characteristics between Obesity and Nonobesity Group among Total Population

\begin{tabular}{llll}
\hline & Obesity & Non-obesity & $P$ value \\
\hline Number & 503 & 5962 & \\
Men, $\mathrm{n}(\%)$ & $225(44.7 \%)$ & $2797(46.9 \%)$ & 0.35 \\
Age, years & $60.21 \pm 12.15$ & $61.2 \pm 12.64$ & 0.09 \\
Systolic blood pressure, $\mathrm{mmHg}$ & $141.89 \pm 19.83$ & $134.99 \pm 21.49$ & $<0.01$ \\
Diastolic blood pressure, $\mathrm{mmHg}$ & $86.67 \pm 10.65$ & $82.74 \pm 11.5$ & $<0.01$ \\
Fasting blood glucose, $\mathrm{mmol} / \mathrm{L}$ & $6.33 \pm 1.54$ & $5.94 \pm 1.21$ & $<0.01$ \\
Triglyceride, mmol/L & $1.97 \pm 1.18$ & $1.48 \pm 1.07$ & $<0.01$ \\
Total cholesterol, mmol/L & $5.18 \pm 0.94$ & $4.95 \pm 0.98$ & $<0.01$ \\
HDL cholesterol, mmol/L & $1.35 \pm 0.34$ & $1.45 \pm 0.38$ & $<0.01$ \\
LDL cholesterol, mmol/L & $2.98 \pm 0.74$ & $2.77 \pm 0.73$ & $<0.01$ \\
Atherogenic index of plasma & $0.13 \pm 0.26$ & $-0.04 \pm 0.28$ & $<0.01$ \\
\hline
\end{tabular}

Note: Variables are expressed as the mean \pm standard deviation. HDL cholesterol, high-density lipoprotein cholesterol; LDL cholesterol, low-density lipoprotein cholesterol 
Table 2 Baseline Characteristics according to Atherogenic Index of Plasma Quartile among Total Population

\begin{tabular}{|c|c|c|c|c|c|}
\hline & Quartile $1<-0.231$ & Quartile $2(-0.231)-(-0.052)$ & Quartile $3(-0.053)-(0.146)$ & Quartile $4 \geq 0.147$ & $P$ value \\
\hline Number & 1614 & 1619 & 1618 & 1614 & \\
\hline Men, $\mathrm{n}(\%)$ & $807(50 \%)$ & $727(44.9 \%)$ & $712(44 \%)$ & $776(48.1 \%)$ & 0.23 \\
\hline Age, years & $63.6 \pm 12.26$ & $62.16 \pm 12.7$ & $60.53 \pm 12.49$ & $58.21 \pm 12.33$ & $<0.01$ \\
\hline Body mass index, $\mathrm{kg} / \mathrm{m}^{2}$ & $21.8 \pm 2.93$ & $22.95 \pm 2.97$ & $23.92 \pm 2.99$ & $25.01 \pm 2.94$ & $<0.01$ \\
\hline Systolic blood pressure, $\mathrm{mmHg}$ & $136.27 \pm 21.52$ & $135.68 \pm 21.95$ & $135.06 \pm 21.55$ & $135.11 \pm 20.74$ & 0.33 \\
\hline Diastolic blood pressure, $\mathrm{mmHg}$ & $82.3 \pm 11.93$ & $82.69 \pm 11.53$ & $83 \pm 11.21$ & $84.18 \pm 11.18$ & $<0.01$ \\
\hline Fasting blood glucose, mmol/L & $5.8 \pm 1.06$ & $5.91 \pm 1.19$ & $5.95 \pm 1.13$ & $6.2 \pm 1.51$ & $<0.01$ \\
\hline Triglyceride, mmol/L & $0.76 \pm 0.18$ & $1.09 \pm 0.21$ & $1.5 \pm 0.33$ & $2.72 \pm 1.54$ & $<0.01$ \\
\hline Total cholesterol, mmol/L & $4.87 \pm 0.88$ & $4.92 \pm 0.95$ & $4.99 \pm 1$ & $5.08 \pm 1.05$ & $<0.01$ \\
\hline $\mathrm{HDL}$ cholesterol, mmol/L & $1.76 \pm 0.38$ & $1.51 \pm 0.28$ & $1.35 \pm 0.26$ & $1.15 \pm 0.3$ & $<0.01$ \\
\hline LDL cholesterol, mmol/L & $2.5 \pm 0.6$ & $2.75 \pm 0.67$ & $2.93 \pm 0.73$ & $2.97 \pm 0.83$ & $<0.01$ \\
\hline
\end{tabular}

Note: Variables are expressed as the mean \pm standard deviation. HDL cholesterol, high-density lipoprotein cholesterol; LDL cholesterol, low-density lipoprotein cholesterol

tended to be younger and have higher BMI, DBP, FBG, TG, TC, LDL-C as well as lower HDL-C.

Figure 1 showed the significant linear correlations between AIP, four lipid profiles and BMI. TG, TC, LDL-C and AIP positively correlated to BMI while HDL-C inversely correlated to it. Among them, AIP showed a strongest association with BMI as the coefficient reached up to $0.372(P<0.01)$.

In Table 3, univariate analyses showed the significant associations between AIP, four lipid profiles and obesity $(P<0.01)$. After adjustment for age, gender, SBP, DBP and FBG, the associations remained significant $(P<$ 0.01). Both in these two models, AIP had a highest OR of obesity compared with the other lipid components. After further adjustment for other lipid components, AIP remained to have a strongest association with obesity. In addition, participants in higher AIP quartiles all had a significantly increased risk of obesity compared with the reference group with the ORs of 2.573, 3.765 and 6.205, respectively ( $P$ for trend $<0.01$ ).

As shown in Fig. 2 and Table 4, compared with the model including TG, HDL-C, age, gender, SBP, DBP, FBG, TC and LDL-C, the model including AIP, age, gender, SBP, DBP, FBG, TC and LDL-C significantly improved the accuracy of risk prediction for obesity. After replacing TG and HDL-C with AIP, the AUC for the

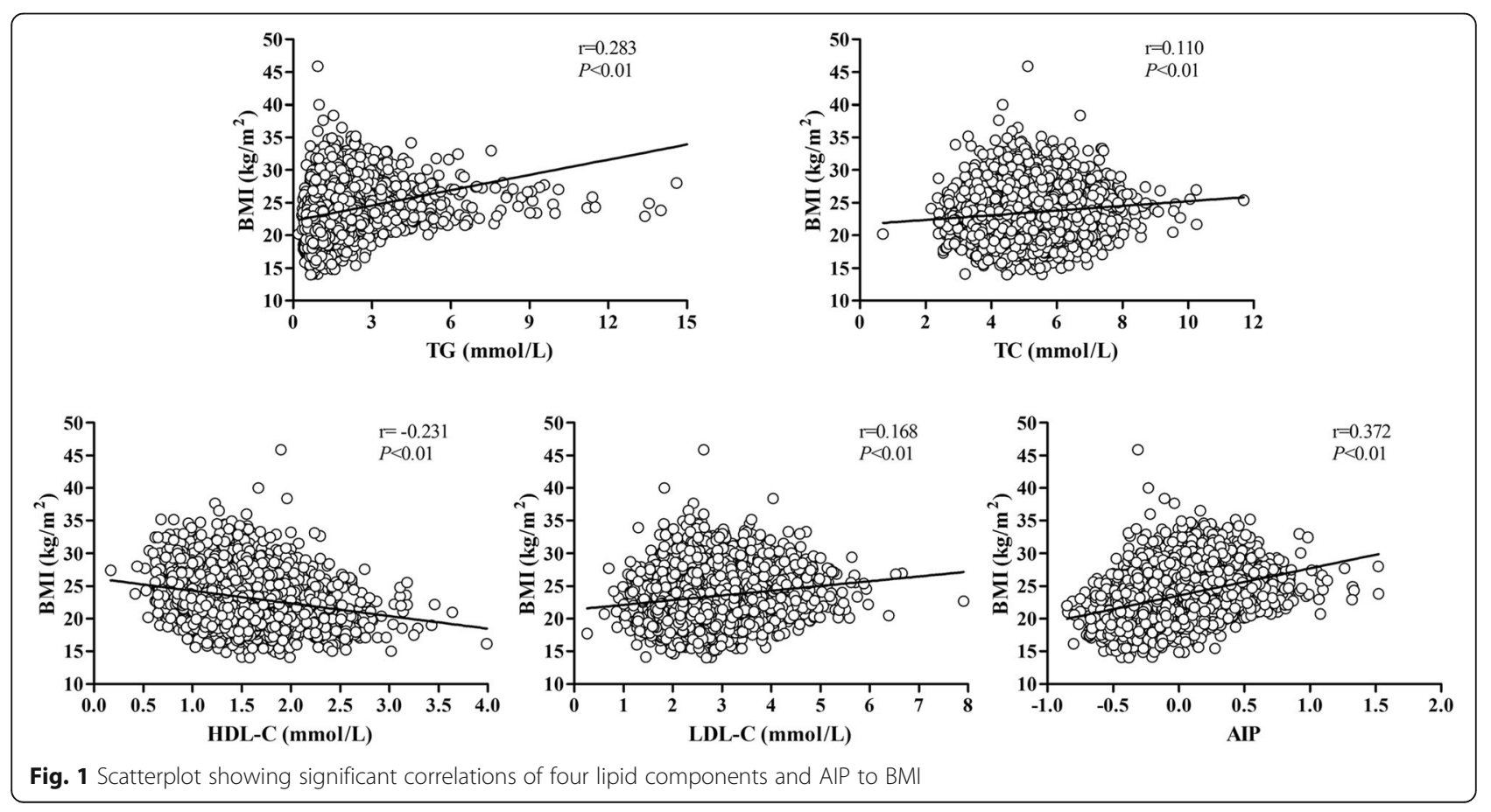


Table 3 Odds Ratios (95\% Confidence Interval) of Obesity according to Lipid Components

\begin{tabular}{|c|c|c|c|c|c|c|}
\hline & unadjusted & $P$ value & multivariable adjusted* & $P$ value & multivariable adjusted ${ }^{* *}$ & $P$ value \\
\hline$\overline{T G}$ & $1.295(1.217-1.378)$ & $<0.01$ & $1.229(1.154-1.308)$ & $<0.01$ & $1.123(1.029-1.226)$ & $<0.01$ \\
\hline TC & $1.253(1.147-1.369)$ & $<0.01$ & $1.196(1.088-1.315)$ & $<0.01$ & $1.207(0.963-1.512)$ & 0.103 \\
\hline $\mathrm{HDL}-\mathrm{C}$ & $0.45(0.348-0.584)$ & $<0.01$ & $0.375(0.283-0.496)$ & $<0.01$ & $0.354(0.233-0.539)$ & $<0.01$ \\
\hline \multirow{2}{*}{$\begin{array}{l}\text { LDL-C } \\
\text { AIP }\end{array}$} & $1.445(1.287-1.622)$ & $<0.01$ & $1.391(1.237-1.566)$ & $<0.01$ & $1.113(0.873-1.420)$ & 0.386 \\
\hline & $6.691(4.956-9.034)$ & $<0.01$ & $5.829(4.272-7.952)$ & $<0.01$ & $5.550(4.03-7.644)$ & $<0.01$ \\
\hline \multicolumn{7}{|l|}{ AIP Quartiles } \\
\hline$<-0.231$ & 1.000(Reference) & & 1.000(Reference) & & 1.000(Reference) & \\
\hline$(-0.231)-(-0.052)$ & $2.732(1.85-4.036)$ & $<0.01$ & $2.672(1.806-3.954)$ & $<0.01$ & $2.573(1.735-3.813)$ & $<0.01$ \\
\hline$(-0.053)-(0.146)$ & $4.152(2.859-6.029)$ & $<0.01$ & $4.014(2.758-5.842)$ & $<0.01$ & $3.765(2.575-5.506)$ & $<0.01$ \\
\hline$\geq 0.147$ & $7.358(5.142-10.53)$ & $<0.01$ & $6.689(4.652-9.618)$ & $<0.01$ & $6.205(4.293-8.968)$ & $<0.01$ \\
\hline$P$ for trend & $<0.01$ & & $<0.01$ & & $<0.01$ & \\
\hline
\end{tabular}

Note. TG, TC, HDL-C, LDL-C and AIP indicate triglyceride, total cholesterol, high-density lipoprotein cholesterol, low-density lipoprotein cholesterol and atherogenic index of plasma. * Multivariable model included age, gender, systolic blood pressure (SBP), diastolic blood pressure (DBP), fasting blood glucose (FBG) and any one lipid component.** Multivariable model included two models. One model included TG, HDL-C, TC, LDL-C, age, gender, SBP, DBP and FBG. In this model, the ORs ( $95 \% \mathrm{Cls}$ ) of TG, TC, HDL-C and LDL-C were calculated. The other model included AIP, TC, LDL-C, age, gender, SBP, DBP and FBG. In this model, the ORs $(95 \% \mathrm{Cls})$ of AIP and its quartiles were calculated

model improved by 0.011 (AUC changed from 0.706 to $0.717 ; P=0.011)$. The continuous and category NRIs were $29.55 \%(P<0.01)$ and $6.06 \%(P<0.01)$, and the IDI was $0.68 \%(P<0.01)$.

\section{Discussion}

In this large-sample cross-sectional survey, our findings indicated that subjects with higher AIP levels tended to have a higher risk of obesity. We also for the first time detected that integrating the two indies (TG and HDL-C) to generate the composite one of AIP is more likely to increase the predictive sensibility for obesity. AIP can be considered as a novel and better biomarker for obesity.

AIP, calculated as $\log _{10}(\mathrm{TG} / \mathrm{HDL}-\mathrm{C})$, was initially constructed as a biomarker of plasma atherosclerosis [6] and has been proved to be significantly correlated with other important atherosclerosis indexes such as LDL-C size and small-dense LDL-C $[11,12]$. To date, quite a few studies have detected an association between AIP and atherosclerosis-related conditions. For example, Abdullah et al. and Cure et al. demonstrated that AIP was significantly and positively correlated with carotid intima-media thicknesses $(\mathrm{r}=0.304, P<0.001 ; \mathrm{r}=0.261$, $P<0.001)[13,14]$. A large-sample case-control study in China also indicated that AIP was significantly associated with coronary artery disease with an adjusted OR (95\%CI) of 1.66 (1.367-2.016) [9]. Although a previous study reported a higher prevalence of obesity associated with AIP elevating, it did not adjust other confounding factors and compare the magnitude of the association between AIP, conventional lipid profiles and obesity [15].

The conventional lipid components are recommended to be used in atherosclerotic cardiovascular diseases $[16,17]$.
Several lipoprotein ratios have been established and widely used in recent years. The balance constructed between atherogenic and protective lipoproteins makes the lipoprotein ratios have greater cardiovascular risk than conventional lipid components used alone [18]. Among those ratios, AIP has been reported to be a better biomarker for coronary artery disease [9] and type 2 diabetes [19] than the conventional lipids. In our study, the Pearson analyses, logistic analyses and especially predictive analyses including AUC, NRI and IDI all indicated that AIP was a useful and superior biomarker




Table 4 Comparison of Discrimination Performance for Obesity between AlP and Conventional Lipid Components including TG and HDL-C Levels

\begin{tabular}{llll}
\hline & NRI (Continuous), $\%$ & NRI (Category), \% & IDI, \% \\
\hline Conventional model adding TG and HDL-C & 1.00 (Reference) & 1.00 (Reference) & $1.00($ Reference) \\
Conventional model adding AIP & $29.55(20.57-38.52)$ & $6.06(3.06-9.07)$ & $0.68(0.5-0.86)$ \\
$P$ value & $<0.01$ & $<0.01$ & $<0.01$
\end{tabular}

Notes: TG, HDL-C and AIP indicate triglyceride, high-density lipoprotein cholesterol and atherogenic index of plasma. The conventional model included age, gender, systolic blood pressure, diastolic blood pressure, fasting blood glucose, total cholesterol and low-density lipoprotein cholesterol

*Patients were divided into three categories for risk classification: $<5 \%, 5 \%-15 \%$, and $>15 \%$. NRI, net reclassification improvement; IDI, integrated discrimination index

for obesity. Enhancing the control of AIP rather than conventional lipids may better prevent obesity prevalence in the total population.

Our study has a few advantages that deserve mentioning. At first, this is a large-scale survey research in an adult population of China, and it proves AIP has the superior predictive power than conventional lipid profiles for obesity for the first time. All information collection and laboratory assay were conducted with strict quality controls. In addition, the findings of a strongest association between AIP and obesity as well as the predictive power of AIP superior to the conventional lipids were stable and reliable because of a high statistically power. The integration of the two indies (TG and HDL-C) to generate a composite one of AIP for obesity could avoid the inconsistent assessment of different lipid components and simplify the prediction task in practical application. Furthermore, as obesity is generally recognized as the important risk factor of many diseases, AIP could be considered as a novel and applicable biomarker for the related diseases incidence and prognosis. Future drug discovery for obesity and related diseases could be conducted with AIP as a treatment target.

There were also some limitations that should be mentioned. First, the feature of cross-sectional study contributed to the difficulty in building the definite causal relationship between blood lipid profiles and obesity. However, it is reasonable for AIP to be an independent risk factor for obesity because of the biological association between blood lipid profiles and obesity. Second, the data of other confounders including diet, medical and drug history, tobacco and alcohol consumption as well as physical activity were not included in this analysis because of the information default. The possibility that these variables confounded the true association between AIP and obesity cannot be completely eliminated. We will include these relevant variables in the further researches and combine them with AIP together in obesity prediction. Third, the levels of lipid profiles in our study were determined with a single measurement. Therefore, some exposure misclassification risk is inevitable but is probably low, as previous work demonstrated that lipid components are stable over time [20].

\section{Conclusions}

Higher AIP level was positively and strongly associated with obesity. AIP is a novel and better biomarker associated with obesity. It can be used in clinical practice as a direct biological target to evaluate the effect of hypolipidemic drugs on the prevention and treatment of obesity, which may facilitate clinicians to adjust the medication plan in order to reduce the adverse reactions caused by long-term use of lipid-lowering drugs.

\section{Abbreviations}

AIP: Atherogenic index of plasma;; AUC: Area under the curve; BMI: Body mass index; DBP: Diastolic blood pressure; FBG: Fasting blood glucose; HDLC: High-density lipoprotein cholesterol; IDI: Integrated discrimination improvement; LDL-C: Low-density lipoprotein cholesterol; NRI: Net reclassification index; OR: odds ratio; ROC: Receiver operating characteristic; SBP: Systolic blood pressure; TC: Total cholesterol; TG: Triglyceride

\section{Acknowledgements}

Not applicable.

\section{Funding}

This study was supported by Natural Science Foundation of China (81473046,31271336, 81373010, 81502868, 31401079, 81401343, 81541068), the Natural Science Foundation of Jiangsu Province (BK20130300, BK20150346) and the Project of Preventive Medicine of Jiangsu Province (Y2012024).

\section{Availability of data and materials}

The datasets during and/or analyzed during the current study are available from the corresponding author on reasonable request.

\section{Authors' contributions}

ZXW researched the data and wrote the manuscript. YLG, ZH, MQH, ZXH, LT, HJR and XWX collected the data. YNJ researched the data. LSF designed the study, collected data and revised the manuscript. All authors read and approved the final manuscript.

\section{Ethics approval and consent to participate}

The study was approved by the ethical committee of Soochow University. Subjects agreeing to participate into the present study provided a written informed consent.

\section{Consent for publication}

Not applicable.

Competing interests

The authors declare that they have no competing interests.

\section{Publisher's Note}

Springer Nature remains neutral with regard to jurisdictional claims in published maps and institutional affiliations. 


\section{Author details}

'Center for Genetic Epidemiology and Genomics, School of Public Health Medical College of Soochow University, Suzhou, Jiangsu 215123, China. 2Jiangsu Key Laboratory of Preventive and Translational Medicine for Geriatric Diseases, School of Public Health, Medical College of Soochow University, Suzhou, Jiangsu 215123, China. ${ }^{3}$ Center for Disease Control and Prevention of Suzhou Industry Park, Suzhou, Jiangsu 215021, China. ${ }^{4}$ No. 3 People's Hospital of Xiang Cheng District, Suzhou, Jiangsu 215134, China. ${ }^{5}$ Department of Biostatistics, University of Alabama at Birmingham, Birmingham, AL 35294, USA.

Received: 17 January 2018 Accepted: 21 February 2018

Published online: 05 March 2018

\section{References}

1. Collaboration NCDRF. Trends in adult body-mass index in 200 countries from 1975 to 2014: a pooled analysis of 1698 population-based measurement studies with 19.2 million participants. Lancet. 2016:387:1377-96.

2. Bays HE, Gonzalez-Campoy JM, Bray GA, Kitabchi AE, Bergman DA, Schorr $A B$, et al. Pathogenic potential of adipose tissue and metabolic consequences of adipocyte hypertrophy and increased visceral adiposity. Expert Rev Cardiovasc Ther. 2008;6:343-68.

3. Bays HE, Toth PP, Kris-Etherton PM, Abate N, Aronne LJ, Brown W, et al. Obesity, adiposity, and dyslipidemia: a consensus statement from the National Lipid Association. J Clin Lipidol. 2013;7:304-83.

4. Nishide R, Ando M, Funabashi H, Yoda Y, Nakano M, Shima M. Association of serum hs-CRP and lipids with obesity in school children in a 12-month follow-up study in Japan. Environ Health Prev Med. 2015;20:116-22.

5. Skinner AC, Perrin EM, Moss LA, Skelton JA. Cardiometabolic risks and severity of obesity in children and young adults. N Engl J Med. 2015;373: 1307-17.

6. Dobiasova M, Frohlich J. The plasma parameter log (TG/HDL-C) as an atherogenic index: correlation with lipoprotein particle size and esterification rate in apoB-lipoprotein-depleted plasma (FER(HDL)). Clin Biochem. 2001:34:583-8.

7. Bora K, Pathak MS, Borah P, Hussain MI, Das D. Association of the Apolipoprotein A-I gene polymorphisms with cardiovascular disease risk factors and Atherogenic indices in patients from Assam, Northeast India. Balkan J Med Genet. 2017:20:59-70.

8. Yang SH, Du Y, Li XL, Zhang Y, Li S, Xu RX, et al. Triglyceride to high-density lipoprotein cholesterol ratio and cardiovascular events in diabetics with coronary artery disease. Am J Med Sci. 2017;354:117-24.

9. Cai G, Shi G, Xue S, Lu W. The atherogenic index of plasma is a strong and independent predictor for coronary artery disease in the Chinese Han population. Medicine (Baltimore). 2017:96:e8058.

10. Zhou B. Coorperative meta-analysis group of China obesity task F. Predictive values of body mass index and waist circumference to risk factors of related diseases in Chinese adult population. Zhonghua Liu Xing Bing Xue Za Zhi. 2002;23:5-10.

11. Kucera M, Oravec S, Hirnerova E, Huckova N, Celecova Z, Gaspar L, et al. Effect of atorvastatin on low-density lipoprotein subpopulations and comparison between indicators of plasma atherogenicity: a pilot study. Angiology. 2014;65(9):794.

12. Ivanova EA, Myasoedova VA, Melnichenko AA, Grechko AV, Orekhov AN Small dense low-density lipoprotein as biomarker for atherosclerotic diseases. Oxidative Med Cell Longev. 2017:2017:1273042.

13. Icli A, Cure E, Uslu AU, Sakiz D, Cure MC, Ozucan M, et al. The relationship between Atherogenic index and carotid artery atherosclerosis in familial Mediterranean fever. Angiology. 2017;68:315-21.

14. Cure E, Icli A, Ugur Uslu A, Aydogan Baykara R, Sakiz D, Ozucan M, et al. Atherogenic index of plasma may be strong predictor of subclinical atherosclerosis in patients with Behcet disease. Z Rheumatol. 2017;76:259-66.

15. Shen SW, Lu Y, Li F, Shen ZH, Xu M, Yao WF, et al. Potential long-term effects of previous schistosome infection may reduce the atherogenic index of plasma in Chinese men. Int J Parasitol. 2015:45:289-94.

16. Stone NJ, Robinson JG, Lichtenstein AH, Bairey Merz CN, Blum CB, Eckel RH, et al. 2013 ACC/AHA guideline on the treatment of blood cholesterol to reduce atherosclerotic cardiovascular risk in adults: a report of the American College of Cardiology/American Heart Association task force on practice guidelines. Circulation. 2014;129:51-45.
17. European Association for Cardiovascular P. Rehabilitation, Reiner Z, Catapano AL, de backer G, Graham I, et al. ESC/EAS guidelines for the management of dyslipidaemias: the task force for the management of dyslipidaemias of the European Society of Cardiology (ESC) and the European atherosclerosis society (EAS). Eur Heart J. 2011;32:1769-818.

18. Millan J, Pinto X, Munoz A, Zuniga M, Rubies-Prat J, Pallardo LF, et al. Lipoprotein ratios: physiological significance and clinical usefulness in cardiovascular prevention. Vasc Health Risk Manag. 2009;5:757-65.

19. Zhu XW, Deng FY, Lei SF. Meta-analysis of Atherogenic index of plasma and other lipid parameters in relation to risk of type 2 diabetes mellitus. Prim Care Diabetes. 2015:9:60-7.

20. Konopka A, Chodkowska E, Piotrowski W, Stepinska J. Is the assessment of lipid profile performed up to seventy two hours from hospital admission due to acute coronary syndrome still valid? Kardiol Pol. 2004;61:243-50. discussion $51-2$

\section{Submit your next manuscript to BioMed Central and we will help you at every step:}

- We accept pre-submission inquiries

- Our selector tool helps you to find the most relevant journal

- We provide round the clock customer support

- Convenient online submission

- Thorough peer review

- Inclusion in PubMed and all major indexing services

- Maximum visibility for your research

Submit your manuscript at www.biomedcentral.com/submit
C) Biomed Central 